\title{
Peering into the heart of the M82 starburst
}

\author{
Danielle Fenech $^{1}$, Tom Muxlow ${ }^{2}$, Rob Beswick ${ }^{2}$ and Megan Argo ${ }^{2}$ \\ ${ }^{1}$ Department of Physics and Astronomy, University College London, Gower Street, London, \\ WC1E 6BT, U.K. email: dmf@star.ucl.ac.uk \\ ${ }^{2}$ Jodrell Bank Centre for Astrophysics, The University of Manchester, U.K.
}

\begin{abstract}
We present multi-epoch millarcsecond resolution images of the most compact supernova remnants in the nearby starburst galaxy M82, spanning 25 years of evolution. In particular, we will briefly discuss the new transient source first detected in 2009 as well as the unusual object $41.95+57.5$ and its potential as a GRB afterglow.
\end{abstract}

Keywords. radio continuum: supernova remnants, techniques: interferometric, high angular resolution

\section{Introduction}

M82 is considered the archetypal starburst galaxy and at a distance of $\sim 3.6 \mathrm{Mpc}$ (Freedman et al. 1994) is one of the closest examples of its kind. It therefore provides a unique opportunity to study a star-forming environment in detail and particularly the discrete products of star-formation such as supernova remnants (SNR) and HII regions. Supernovae and supernova remnants play an important role in the feedback of energy and material into the surrounding interstellar medium as evidenced in M82 by the galactic superwind driven by the numerous supernovae, SNR and massive stellar winds. Radio observations can be used to see into the core of the star-forming region in the centre of M82 as they are unaffected by the gas and dust associated with such an intense starburst environment. Since their discovery in the 1970 s, radio observations have been used to study and monitor the evolution of the $\sim 100$ supernova remnants at the heart of this galaxy (e.g. Unger et al. 1984, Kronberg, Biermann \& Schwab 1985, Muxlow et al. 1994, Fenech et al. 2005).

\section{Milliarcsecond resolution monitoring}

The young SNR within M82 are very compact ( few tens of mas) and require high angular resolution observations in order to resolve their structure. Early observations (1986; 15 mas resolution) using the EVN (European VLBI Network) studied the then brightest and most compact source, 41.95+57.5. Further EVN and global VLBI observations of M82 in 2015 follow multiple epochs of observations made since 1986. These form the latest epoch of an ongoing radio monitoring campaign studying the evolution of the most compact radio SNR in the core region of M82 (see McDonald et al. 2001, Beswick et al. 2006, Fenech et al. 2010). This selection has now expanded to include four SNR, SN2008iz and a transient source, whose nature is still unknown.

$41.95+57.5$ - a possible GRB remnant? This is a peculiar source in the centre of M82, which dominated the flux density profile of the whole galaxy during the first observations in 1960 Muxlow et al. (2005). The monitoring observations of this source have displayed atypical behaviour for a Type II SNR. The flux density of $41.95+57.5$ has been decaying at a rate of $\sim 8.5 \% \mathrm{yr}^{-1}$ (Muxlow et al. 2005, Gendre et al. 2013). Furthermore it has a distinct bi-polar morphology on the scale of $\sim 20$ mas. Monitoring of the expansion of 
the two peaks of emission have determined velocities of $\sim 1500-2000 \mathrm{kms}^{-1}$ (Beswick et al. 2006, Fenech et al. 2010). If it is assumed to be in free expansion this source will be $\sim 100$ yrs old (it is at least $60-65$ yrs old from early M82 flux measurements). Extrapolating the flux density decline of this source back implies it would have peak luminosity at $5 \mathrm{GHz}$ of $\sim 2 \times 10^{30} \mathrm{ergss}^{-1} \mathrm{~Hz}^{-1}$. This would be significantly brighter than the brightest Known Type II SN and approaching the luminosity of long-duration gamma-ray burst.

The 'transient' source 43.78+59.3 This source appeared in early 2009 with a flux density of $\sim 0.7 \mathrm{mJy}$ at $5 \mathrm{GHz}$. It had a rapid turn-on with a steep spectral index. This source increased in flux density to around $1 \mathrm{mJy}$ after around 400 days and has remained approximately constant since, though within the last year has now started to decline. Muxlow et al. (2010) offered several scenarios to explain the nature of this source including a peculiar SN, a microquasar and IMBH. However these do not fully explain the observed characteristics. Muxlow et al. (2010) also detected a tentative superluminal motion in an East-West direction. Global VLBI 5 GHz 1 mas resolution observations of this source show an extended structure in the direction of the potential superluminal motion.

\section{References}

Beswick, R. et al. 2006, MNRAS, 369, 1221

Fenech, D., Muxlow, T., Beswick, R., Pedlar, A., \& Argo, M. 2008, MNRAS, 391, 1384

Fenech, D., Beswick, R., Muxlow, T., Pedlar, A., \& Argo, M. 2010, MNRAS, 408, 607

Freedman, W. et al. 1994, ApJ, 427, 241

Gendre, M., Fenech, D., Beswick, R., Muxlow, T. \& Argo, M. 2013 MNRAS, 431, 1107

Kronberg, P., Biermann, P., \& Schwab, F. 1985, ApJ, 291, 693

McDonald, A., Muxlow, T., Pedlar, A., Garrett, M., Wills, K., Garrington, S., Diamond, P., \& Wilkinson, P. 2001, MNRAS, 322, 100

Muxlow, T., Pedlar, A., Wilkinson, P., Axon, D., Sanders, E., \& de Bruyn, A. 1994, MNRAS, 266,455

Muxlow, T., Pedlar, A., Beswick, R., Argo, M., O'Brien, T., Fenech, D., \& Trotman, W. 2008, MmSAI, 76, 586

Muxlow, T. et al. 2008, MNRAS, 404, 109

Unger, S., Pedlar, A., Axon, D., Wilkinson, P., \& Appleton, P. 1984, MNRAS, 211, 783 